\title{
Potential of cell-free supernatants from cultures of selected lactic acid bacteria and yeast obtained from local fermented foods as inhibitors of Listeria monocytogenes, Salmonella spp. and Staphylococcus aureus
}

Solomon H Mariam ${ }^{1,2^{*}}$, Nigus Zegeye ${ }^{2,3}$, Tewodros Tariku², Emawayish Andargie ${ }^{2}$, Nigatu Endalafer ${ }^{2}$ and Abraham Aseffa ${ }^{2}$

\begin{abstract}
Background: Food-borne infections cause huge economic and human life losses worldwide. The most common contaminants of foods include Listeria monocytogenes Salmonellae and Staphylococcus aureus. L. monocytogenes is most notorious due to its tolerance to common food preservation methods and the risks it poses, including higher fatality rates. Safer, more efficacious control methods are thus needed. Along with food-borne pathogens, lactic acid bacteria $(L A B)$ can also be found in foods. Some $L A B$ isolates inhibit pathogenic bacteria by various mechanisms, including by production of antimicrobial metabolites.

Methods: The potential of cell-free culture supernatants (CFS) derived from broth cultures of selected local LAB and yeast isolates, some of which were subjected to various treatments, were tested for inhibition of L. monocytogenes, Salmonella spp. and S. aureus in in vitro culture by incorporating various proportions of the CFSs into the growth medium concurrently with inoculation (co-cultures) or following limited proliferation after inoculation of the pathogens (delayed cultures). The effects of the CFSs on various growth parameters were assessed.

Results: CFS from the LAB isolates were strongly inhibitory when co-cultured. The inhibitory activities were stable following heat or protease treatment of the CFSs. Inhibitory activity was dependent primarily on active substance(s) secreted into the supernatant. In all co-cultures, CFS proportion-dependent progressive decrease in the number of colonies was observed and both growth rates and number of generations were reduced with significantly fewer numbers of colony forming units, whereas generation times were significantly increased compared to those of controls. Transfer from co-cultures to fresh broth showed inhibited cultures contained bacteria that can re-grow, indicating the presence of viable bacteria that are undetectable by culture. Growth rates in CFS-treated delayed cultures were also reduced to varying degrees with the number of colonies in some cultures being significantly less than the corresponding control values. CFSs were active against both Gram-positive and -negative bacteria.

(Continued on next page)
\end{abstract}

\footnotetext{
* Correspondence: solomon.habtemariam@aau.edu.et

'Aklilu Lemma Institute of Pathobiology, Addis Ababa University, Addis Ababa, Ethiopia

${ }^{2}$ Armauer Hansen Research Institute, P.O. Box 1005, Addis Ababa, Ethiopia

Full list of author information is available at the end of the article
} 
(Continued from previous page)

Conclusions: Active metabolites produced and secreted by LAB into the growth medium were effective in inhibiting the tested pathogens. Early addition of the CFSs was necessary for significant inhibition to occur. Further studies will help make these findings applicable to food safety.

Keywords: Lactic acid bacteria, Listeria monocytogenes, Salmonella, Staphylococcus aureus, Inhibition, Cell-free supernatant, Inhibition

\section{Background}

Food-borne infections cause huge economic losses, in addition to morbidity and mortality. Listeria monocytogenes and nontyphoidal Salmonella enterica ranked among the top five identifiable pathogens causing these human and economic losses [1]. A study estimated an aggregate annual cost of 50-77 billion due to food-borne illness in the United States [2]. Staphlococcus aureus also ranked among the top five pathogens that caused foodborne illnesses in 2011 [3].

L. monocytogenes is exceptional in that it can tolerate high salt concentrations, remains viable at temperatures as low as $0^{\circ} \mathrm{C}$, and is also difficult to eliminate by ordinary disinfectants $[4,5]$. It can be found as a contaminant in meat, milk and other food-processing facilities. Risk groups in humans include immuno-compromised persons and the elderly. The infection causes septicemia, meningitis, encephalitis or abortion or stillbirth of neonates, while gastroenteritis can occur in the general public [6]. The infectious dose is unknown but the case fatality rate has been reported to be $20-40 \%$ [6,7], which is much higher than the fatality rate of salmonellosis, the incidence rate of which is, in turn, much higher than listeriosis [6,7].

As well as food-borne pathogens, lactic acid bacteria (LAB) can also be found in various habitats including in foods and drinks of plant and animal origins. Fermented foods and drinks are particularly rich in their content of LAB. LAB species of practical importance are usually nonpathogenic and safe, at least in healthy and immunocompetent people. Several reports have indicated that the shelf-life of foods can be extended by LAB or LAB metabolites [8-11]. Food safety is enhanced because of the inhibitory actions of LAB on common food-borne pathogens such as Escherichia coli O157::H7, Salmonella spp. and Listeria monocytogenes. Inhibition of foodborne pathogens by LAB can be mediated by competitive exclusion or production of organic acids and antimicrobial products such as hydrogen peroxide and antimicrobial peptides (e.g., bacteriocins) [12-14]. These products can be produced by some LAB and secreted when grown in appropriate broth medium. Either live LAB or their metabolic products can be used [14]. Moreover, the antimicrobial properties of some LAB or their antimicrobial peptide products as well as their vaccine potential may make them suitable candidates as bio-therapeutic agents for human and animal applications [15-20]. Thus, selected LAB species with these and additional qualities (termed probiotics, defined as live microorganisms which, when administered in adequate amounts confer health benefit on the host [21]) have been developed.

In this work, we focused on the inhibitory effects of metabolites of a collection of LAB and yeast isolates obtained from local fermented foods and milk on some common food-borne pathogens or standard strains. The choice of these fermented foods and milk is based on the fact that these are consumed regularly by the population. The inhibitory effect of cell-free culture supernatants (CFS) from broth cultures of the selected isolates on growth rates and colony-forming units of L. monocytogenes, Salmonella and S. aureus were tested following various treatments of the CFS as described below.

\section{Methods}

\section{Bacterial isolates and growth conditions}

The sources of LAB used in this study were fermented milk, ananas and a grain called Teff, which are important fermented foods consumed regularly in Ethiopia. The fermentates were diluited in sterile PBS buffer, $\mathrm{pH} 7.2$ and, to obtain single colonies, $100 \mu \mathrm{L}$ of the sixth and seventh 10-fold serial dilutions were plated on De Man, Rogosa, Sharpe (MRS) agar, followed by picking single colonies at random and growing in MRS broth. Incubations of both agar and broth cultures were at $37^{\circ} \mathrm{C}$ at aerobic or anaerobic conditions. Combinations of standard tests (microscopy, cultural and biochemical) [22,23] were used to tentatively identify the isolates. Cell pellets from the MRS broth cultures were resuspended in MRS broth containing 15\% glycerol and aliquots were frozen for use when needed. All media used here were from OXOID, UK. In addition, while we were searching for a LAB isolate from the ananas fermentate, a non-LAB isolate that was found and later identified to be a yeast was used in the inhibition studies.

The bacteria tested for inhibition by the CFSs (target strains) were Listeria monocytogenes (ATCC 19115), Salmonella enterica subsp. enterica serovar Enteritidis (ATCC 13076) and S. aureus (ATCC 25923), all type strains. Another bacterial isolate used was MI1, an isolate 
from bovine milk identified to be Salmonella spp. by tests including Gram staining, colonial morphology, motility and growth on differentiating and selective media (MacKonkey, XLD and Salmonella-Shigella agars), sugar fermentation test with Biomerioux API20 (Biomerioux, France) in comparison with the known properties of Salmonella spp. The results of none of these tests conducted on the milk isolate were similar to those of the known properties of the possible contaminants of milk, such as S. aureus, Listeria, E. coli, Brucella, Campylobacter or Mycobacterium spp. [22,23].

L. monocytogenes was grown in tryptic soy broth (TSB) or tryptic soy agar (TSA). Salmonellae and S. aureus were grown in Luria Bertani (LB) broth or agar. When applicable, agar media were supplemented with $0.5 \%$ pyruvate to enhance detection of possible presence of viable but nonculturable organisms.

\section{Growth of strains for preparation of CFS}

Seventeen isolates from the above sources were grown in MRS broth as described above. The supernatant was recovered by centrifugation at $3000 \mathrm{rpm}$ in a Beckman Allegra 6R (Beckman Coulter) centrifuge for $1 \mathrm{hr}$. CFSs were obtained by passing the supernatants through $0.4 \mu \mathrm{m}$ pore size filters and tested for inhibitory effect on the tested bacteria (in co-cultures, please see below) before adjusting the $\mathrm{pH}$ of the CFSs. Four isolates (total LAB and Lactococcus sp. [both isolated from fermented milk], Streptococcus thermophilus [isolated from Teff] and a yeast species [isolated from ananas]) whose CFSs inhibited the bacteria when tested individually were selected based on this initial observation. These four isolates were grown individually from frozen stock samples in $20 \mathrm{~mL}$ MRS broth and incubating aerobically or anaerobically at $37^{\circ} \mathrm{C}$. In addition, a cocktail made of a mixed culture of these four isolates (Labmix) was also grown in the same way as for the individual isolates. After $18 \mathrm{~h}$ of incubation, an aliquot of $100 \mu \mathrm{L}$ was transferred into $250 \mathrm{~mL}$ of MRS broth and incubated for $24 \mathrm{~h}$. After centrifugation as above, each supernatant was then divided into two portions and subjected successively to the following treatments, further dividing each supernatant into two in going from one treatment to the next.

\section{pH adjustment}

The $\mathrm{pH}$ of the supernatants before $\mathrm{pH}$ adjustment were in the range of 4.5-4.8. A portion of each supernatant was subject to $\mathrm{pH}$ adjustment to $6.5-6.8$.

\section{Proteinase $\mathrm{K}$ treatment of supernatant}

Portions of supernatants were treated with proteinase $\mathrm{K}$ (BDH Biochemicals) at a concentration of $50 \mu \mathrm{g} \mathrm{mL}$ for $1 \mathrm{hr}$ at $37^{\circ} \mathrm{C}$ in a reaction mixture containing $0.5 \%$
SDS, $0.01 \mathrm{M}$ Tris and $0.005 \mathrm{M}$ EDTA [24]. Following treatment, the enzyme was inactivated by addition of phenylmethylsulfonyl fluoride. The supernatants were filtered through $0.4 \mu \mathrm{m}$ pore size filter.

\section{Heat Treatment of CFS}

To test if inhibitory activity was heat-stable or -sensitive, portions were heat-treated $\left(100^{\circ} \mathrm{C}\right.$ for $\left.1 \mathrm{hr}\right)$.

Thus, for each isolate, four types of CFS derived from it were tested for inhibitory activity: both $\mathrm{pH}$-adjusted and heat-treated (CFS1), pH-adjusted only (CFS2), heattreated only (CFS3), and neither $\mathrm{pH}$-adjusted nor heat treated (CFS4). CFS prepared as above was aliquoted and stored at $-20^{\circ} \mathrm{C}$. A fresh aliquot was thawed and used for experiments.

In addition to MRS broth, LAPTg broth was also used to grow the LAB isolates and obtain the CFS. Catalase treatment of the CFSs from LAPTg broth was also conducted to test for possible involvement of hydrogen peroxide in inhibition which, if present, would be abolished by the catalase tretament.

\section{Test of CFS inhibitory effect}

The CFSs were initially tested on the test bacteria L. monocytogenes, Salmonellae and S. aureus by adding CFS to $12.5 \%, 25 \%$ or $50 \%$ proportions (v/v) in $5 \mathrm{~mL}$ final volume. Intermediate CFS proportions were also included as deemed necessary. In either case, $1 \mu \mathrm{L}$ each of $L$. monocy-

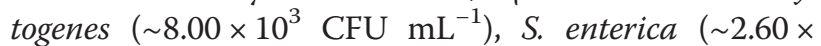
$\left.10^{5} \mathrm{CFU} \mathrm{mL}^{-1}\right)$, Salmonella (MI1) $\left(\sim 1.32 \times 10^{4} \mathrm{CFU} \mathrm{mL}^{-1}\right)$ or $S$. aureus $\left(\sim 1.30 \times 10^{4} \mathrm{CFU} \mathrm{mL}^{-1}\right)$ from frozen stocks were inoculated. The CFSs were added in two different modes to make the $5 \mathrm{~mL}$ final volume according to their percentage proportions: (i) Co-cultures, in which CFS were added simultaneously with inoculation of the test bacteria; (ii) Delayed cultures, in which broth media were inoculated and incubated, followed by CFS addition after 6 hrs of incubation. To obtain the rate of growth of the target strains the turbidity was measured using an optical density of $600 \mathrm{~nm}$ with a spectrophotometer Novaspec II (Pharmacia Biotech) as well as by plating $1 \mu \mathrm{L}$ of 10 -fold serial dilutions of (i) the cocultures at time points day 0 (immediately after both inoculation and CFS addition) and 3 days and (ii) the delayed cultures at time points day 0 (immediately after CFS addition, i.e., after 6 hrs of inoculation and incubation) and 1.5 days. Growth controls for co-cultures included cultures in $5 \mathrm{~mL}$ broth volumes without added CFS. Cultures in which MRS broth was added instead of CFS (to $50 \% \mathrm{v} / \mathrm{v}$ ) were also included. Colonies were usually enumerated after 24 or 48 hrs on TSA agar (L. monocytogenes) or LB agar (Salmonellae and S.aureus).

When no growth was obtained, a sample was withdrawn and inoculated into fresh TSB or LB broth without added 
CFS and growth monitored as for the CFS-treated cultures. In addition, appearance of colonies was assessed by plating 10 or $50 \mu \mathrm{L}$ samples to see if there were surviving cells that were detectable by culture.

\section{Determination of growth rates, generation times, and number of generations}

CFS that showed strong inhibition was selected for each test bacterium and added at the lower inhibitory proportion (30\% for L. monocytogenes, 20\% for S.aureus and $20 \%$ for Salmonella MI1, and S. enterica). Then, rate of growth was quantified by both turbidity measurement and plating on agar media at two hr intervals starting from one hr after incubation of inoculated cultures and continuing during the exponential growth phase. The generation times $(G)$ were calculated from the relationship $G=\ln 2 / k$, where,

$$
k(\text { growth rate })=\left[\left(\log N_{1}-\log N_{0}\right)(2.303)\right] / \mathrm{T}_{1}-\mathrm{T}_{0},
$$

with $\mathrm{N}_{1}$ being the number of bacteria at time $\mathrm{T}_{1}$ (endpoint) and $\mathrm{N}_{0}$ being the number of bacteria at time $T_{0}$ (start of logarithmic growth phase). Number of generations $(\mathrm{N})$ was obtained from:

$$
\mathrm{N}=\left(\log \mathrm{N}_{1}-\log \mathrm{N}_{0}\right) / \log 2 .
$$

Each experiment was conducted three independent times and data represent the averages of these experiments. The Wilcoxon Rank Sum test was used to determine if significant differences exist between means of bacterial numbers (CFUs) of control and experimental cultures.

\section{Test of MRS effect}

To test if any of the components of the pure MRS broth itself could have role in the inhibition, fresh MRS broth was added to the TSB or LB broth to $50 \%$ (v/v) proportion (in co-cultures) and inoculated with the target bacteria and the growth compared to those of the controls (i.e., those grown in $100 \%$ TSB or LB).

\section{Results}

The $\mathrm{pH}$ of the CFSs before $\mathrm{pH}$ adjustment were in the range of 4.5-4.8. Only CFS3 and CFS4 obtained from the four selected isolates and the Labmix exhibited inhibitory activity; i.e., $\mathrm{pH}$-adjusted CFSs were ineffective in inhibition. Inhibitory effect was not abolished by treatment with the protease. The selected isolates were tentatively identified as total LAB and Lactococcus sp. (both isolated from fermented milk), Streptococcus thermophilus (isolated from Teff) and a yeast species isolated from ananas.

\section{Effect of CFS on growth rates and generation times}

In the presence of CFS, both growth rates and number of generations were significantly reduced when compared to those of the controls. Thus, the growth rates of L. monocytogenes, MI1 and S. aureus experimental cultures were reduced by $40 \%, 38 \%$ and $32 \%$ respectively in comparison to those of their respective controls. Accordingly, the number of generations of the experimental cultures decreased by the same percentage points as did the growth rates $(P<0.05)$. Conversely, the generation times of experimental cultures of the three bacteria were significantly increased compared to their respective controls (by 164\%, 162\% and 147\% respectively). Overall, MI1 (the milk isolate) had the highest growth rate, the shortest generation time and the highest number of generations among the three bacteria in both the control and experimental conditions (Table 1).

\section{Simultaneous cultures}

Plating $1 \mu \mathrm{L}$ of dilutions of the L. monocytogenes cocultures on day 3 from the $50 \%$ CFS proportions of total LAB, Lactococcus and Labmix gave no colonies, but after plating $50 \mu \mathrm{L}, 2.26 \times 10^{2} \mathrm{CFU} \mathrm{mL} \mathrm{m}^{-1}$ was obtained from the Lactococcus CFS co-culture only. However, on day 3, the average CFU from the control cultures was $1.71 \times$ $10^{9} \pm 4.77 \times 10^{8}$, an increase of 4.72 Log units over that of day 0 control CFU. CFS from $S$. thermophiles and yeast were less effective against $L$. monocytogenes but nonetheless, the CFU obtained were significantly less than the control $(\mathrm{P} \leq 0.05)$ (Table $2 \mathrm{~A})$.

The $S$. enterica co-cultures exhibited the most dramatic reductions in CFU by day 3 , and this was obtained with the lowest CFS proportions (12.5\%). The $12.5 \%$ proportions of all CFSs caused a $>99 \%$ reduction in CFU compared to the day 3 control CFUs, while the latter increased by $>140$ fold over that of day 0 CFU (i.e., an increase of 2.15 Log units (Table 2B).

The control co-culture of MI1 continued to grow into day 3 of culture by an increase of $4.47 \log$ units relative to that of day 0 control $\left(2.06 \times 10^{9} \pm 1.58 \times 10^{8}\right.$ vs $7.05 \times$ $\left.10^{4} \pm 2.64 \times 10^{3}\right)$. Among the $12.5 \%$ CFS-treated cultures, Labmix and $S$. thermophilus caused the highest reductions in CFU, by 4.83 and $3.83 \mathrm{Log}_{\mathrm{CFU} \mathrm{mL}} \mathrm{mL}^{-1}$ units respectively, relative to the day 3 control $(P \leq 0.05)$. With the highest CFS proportions tested (25\%), the CFU were reduced to zero or to below the level of the day zero CFUs except for that of the yeast CFS (Table 3A). Since zero CFU were obtained for some of the samples with $25 \%$ CFS proportions, increased volumes (10 or $50 \mu \mathrm{L}$ instead of the usual $1 \mu \mathrm{L}$ ) were subsequently plated on day 5 for those cultures which gave zero CFU on day 3 . The result was that either the colonies so obtained were extremely small compared to those from the control or still no CFU were obtained. 
Table 1 Growth rates and generation times and numbers of bacteria in control (CFS-free) and experimental (CFS-treated) cultures

\begin{tabular}{|c|c|c|c|c|c|c|}
\hline \multirow[t]{2}{*}{ Bacteria } & \multicolumn{2}{|c|}{ Growth rate $\left(k, h^{-1}\right)$} & \multicolumn{2}{|c|}{ Generation time (minutes) } & \multicolumn{2}{|c|}{ No of generations } \\
\hline & Control & Exp. & Control & Exp. & Control & Exp. \\
\hline L. monocytogenes & $1.9 \pm 0.002$ & $1.15 \pm 0.006$ & $21.8 \pm 0.025$ & $35.9 \pm 0.004$ & $10.97 \pm 0.013$ & $6.65 \pm 0.03$ \\
\hline Salmonella (Ml1) & $2.9 \pm 0.03$ & $1.8 \pm 0.015$ & $14.3 \pm 0.14$ & $23.2 \pm 0.20$ & $16.73 \pm 0.17$ & $9.83 \pm 0.59$ \\
\hline S. aureus & $1.64 \pm 0.008$ & $1.12 \pm 0.09$ & $25.4 \pm 0.13$ & $37.4 \pm 3.19$ & $9.46 \pm 0.033$ & $6.45 \pm 0.55$ \\
\hline
\end{tabular}

(Exp. = Experimental).

Increased reductions in CFU with increased CFS proportions were also seen with $S$. aureus co-cultures, exhibiting the highest reductions at the 25\% CFS, but even the $12.5 \%$ CFS also caused significant reductions in CFU at day 3 relative to that of the day 3 control $(P \leq 0.05)$ (Table 3B). Comparing CFUs obtained from corresponding CFS proportions, the $12.5 \%$ and $25 \%$ total LAB CFSs resulted in 2.05 and 4.19 Log decreases respectively on day 3 relative to the day 3 control; while that of the control increased by $4.21 \mathrm{Log}$ units relative to the initial, day 0 control. Similarly, the 12.5 and 25\% CFS of Lactococcus caused 1.24 and 3.09 Log units decrease relative the day 3 control CFU.

\section{Delayed cultures}

The L. monocytogenes delayed culture was more resistant to the CFSs than the other bacteria. However, it was kept to at least below the control CFU level by day 1.5 and most cultures gave CFU that were significantly less than the control $(P \leq 0.05)$ (Table $4 \mathrm{~A})$. There was no
CFS proportion-dependent progressive decrease in CFU of $L$. monocytogenes delayed cultures.

The $S$. aureus delayed cultures were insensitive to the tested CFSs at 20\%. However, all CFSs at 25\% reduced the CFU significantly, except that of total LAB $(P \leq 0.05)$ (Table 4B).

With the Salmonella MI1 delayed cultures, day 1.5 CFU from treated cultures were less than the day 1.5 control by 1.07 - 1.47 Log units. (Table 4C), i.e., all day 1.5 experimental CFUs were significantly less than the day 1.5 control $(P \leq 0.05)$.

There was no CFS inhibitory effect on growth of $S$. enterica delayed cultures as there was no difference at all in CFUs between those grown in LB broth with or without any of the CFSs.

\section{MRS effect}

For all target bacteria, there was no difference in the number of CFU obtained in the co-cultures containing $50 \%$ MRS broth (i.e., $50 \%$ MRS $+50 \%$ TSB or LB)

Table 2 Log CFU mL ${ }^{-1}$ of CFS-treated L. monocytogenes (A) and S. enterica (B) co-cultures

\begin{tabular}{|c|c|c|c|c|c|c|}
\hline \multicolumn{7}{|c|}{ A. L. monocytogenes } \\
\hline \multirow[t]{2}{*}{ Day } & CFS (\%) & \multicolumn{5}{|c|}{ CFS } \\
\hline & & Total LAB & Lactococcus & Labmix & \multirow{2}{*}{ S. thermophilus } & Yeast sp. \\
\hline 0 & 0 & \multicolumn{4}{|c|}{$4.00 \times 10^{4} \pm 2.64 \times 10^{3}$} & \\
\hline \multirow[t]{3}{*}{3} & 0 & \multicolumn{5}{|c|}{$1.71 \times 10^{9} \pm 4.77 \times 10^{8}$} \\
\hline & 25 & $1.90 \times 10^{8} \pm 1.13 \times 10^{8 \oplus}$ & $8.00 \times 10^{7} \pm 2.83 \times 10^{7}$ & $1.50 \times 10^{8} \pm 1.41 \times 10^{7}$ & $3.60 \times 10^{7} \pm 1.41 \times 10^{6}$ & $1.82 \times 10^{7}$ \\
\hline & 50 & $0.0^{\Omega}$ & $2.26 \times 10^{2} \pm 3.05 \times 10^{1}$ & $0.0^{\Omega}$ & $6.25 \times 10^{4} \pm 1.06 \times 10^{4}$ & $6.80 \times 10^{7} \pm 2.83 \times 10^{6}$ \\
\hline \multicolumn{7}{|c|}{ B. S. enterica } \\
\hline \multirow[t]{2}{*}{ Day } & CFS (\%) & \multicolumn{5}{|c|}{ CFS } \\
\hline & & Total LAB & \multicolumn{2}{|r|}{ Labmix } & \multicolumn{2}{|l|}{ S. thermophilus } \\
\hline 0 & 0 & \multicolumn{5}{|c|}{$1.30 \times 10^{6} \pm 1.00 \times 10^{5}$} \\
\hline \multirow[t]{2}{*}{3} & 0 & \multicolumn{5}{|c|}{$1.83 \times 10^{8} \pm 1.15 \times 10^{7}$} \\
\hline & 12.5 & $2.50 \times 10^{2} \pm 7.0 \times 10^{1}$ & $5.00 \times 10^{4} \pm 0.0^{\Sigma}$ & $4.20 \times 10^{3} \pm 4.24 \times 10^{2}$ & $8.40 \times 10^{3} \pm 5.65 \times 10^{2}$ & ND \\
\hline \multicolumn{7}{|c|}{ 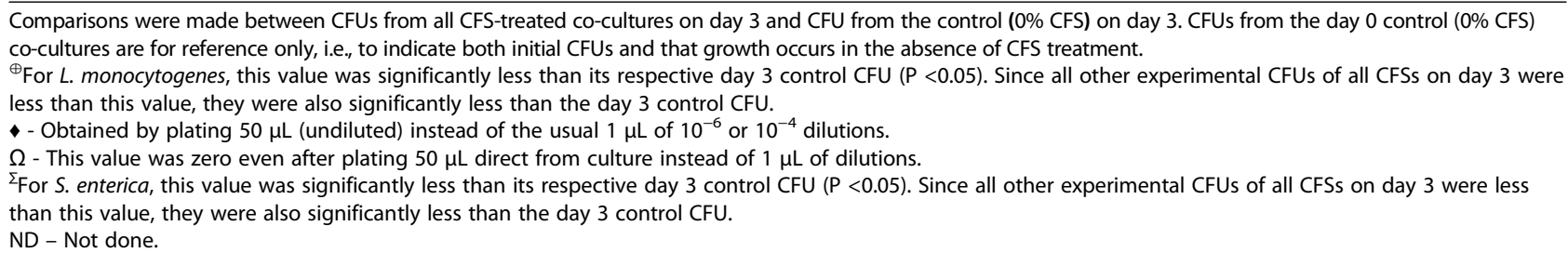 } \\
\hline
\end{tabular}


Table 3 Log CFU $\mathrm{mL}^{-1}$ of CFS-treated Salmonella (MI1) (A) and S. aureus (B) co-cultures

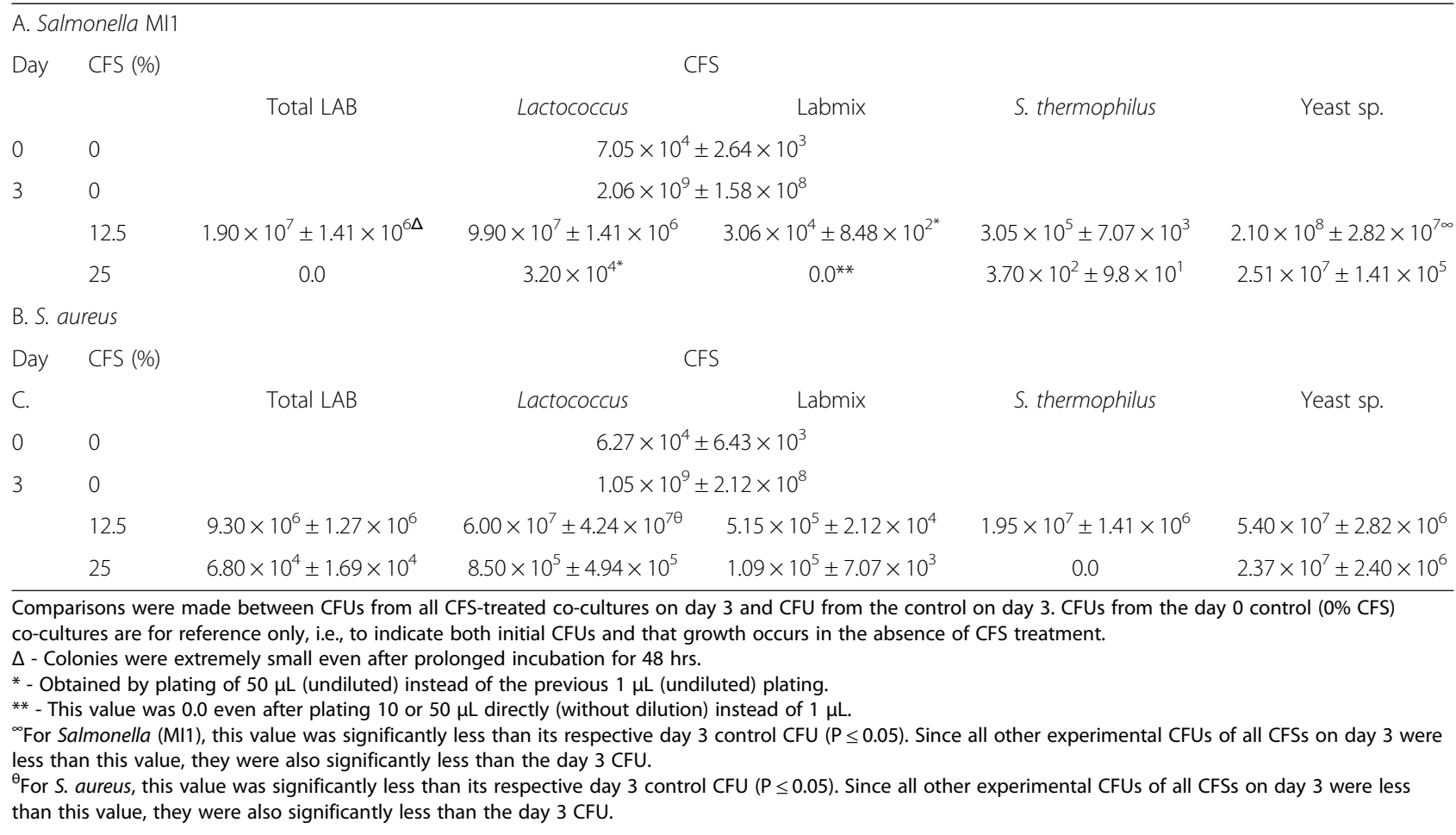

compared to the controls (i.e., cultures in $100 \%$ TSB or LB).

\section{Discussion}

In this work, CFSs derived from selected LAB and yeast broth cultures were tested for inhibitory effect on various bacteria in cultures to which CFS were added in simultaneous or delayed mode. Inhibitory effect was much stronger in co-cultures. All test bacteria were inhibited at all CFS proportions tested in co-cultures, with stronger inhibition at the higher CFS proportions. The inhibition of $S$. enterica in co-culture is particularly noteworthy for two reasons: the initial (day 0) CFU was the highest among those of all the target bacteria and the inhibition was exerted by the lowest CFs proportion.

The delayed cultures showed that even with late addition of CFS, it was possible to retard the growth, in most cases, to at least below the control level at the end of the experiment.

The inhibitory action of the CFSs was not abolished by treatments with proteinase $\mathrm{K}$. The inhibitory substance (s) in the CFSs were heat-resistant as activity was not abolished after heat treatment. They do not appear to be of the class III non-bacteriocin-like lytic proteins $[25,26]$ due to their heat resistance. They were also equally active against both Gram-positive and -negative bacteria. Bacteriocin-like, heat-stable substances active against either or both groups of bacteria have been described [27-30]. These results suggest the non-protein (i.e., nonbacteriocin) nature of the active substances within the CFSs. Treatment with catalase did not result in abolition of activity either, which might indicate hydrogen peroxide was not responsible for the observed activities. There have been some reports about the unsuitability of MRS broth for growth and production of LAB CFS to be used in inhibition of certain pathogens due to its inhibition of production of hydrogen peroxide [31,32]. The recommended medium instead was LAPTg broth. Accordingly, this broth was used here to grow LAB and obtain the CFS. The CFS so obtained did not result in enhanced inhibition of L. monocytogenes or Salmonellae or S. aureus. In fact, growth of these bacteria in CFS obtained from LAPTg broth-grown LAB was equal to or slightly higher than CFS from MRS broth-grown LAB. These results may be suggestive of a minor, if any, role for $\mathrm{H}_{2} \mathrm{O}_{2}$ in the observed inhibition in MRS broth CFS. Catalase treatment of the CFS obtained from the LAPTg broth culture did not result in enhanced growth of the target bacteria either. In addition, $\mathrm{H}_{2} \mathrm{O}_{2}$ in the CFSs would have had little, if any, role in inhibition, at least in the cases of L. monocytogenes and S. aureus, as these organisms are catalase producers themselves. Both of these findings (the non-protein and non- $\mathrm{H}_{2} \mathrm{O}_{2}$ nature of the active substances) bear an interesting similarity to the results of Bleicher et al. [33]. The inhibition observed in 
Table 4 Log CFU mL $^{-1}$ of CFS-treated L. monocytogenes (A), S. aureus (B) and Salmonella (MI1) (C) delayed cultures

\begin{tabular}{|c|c|c|c|c|c|}
\hline \multicolumn{6}{|c|}{ A. L. monocytogenes } \\
\hline \multirow[t]{2}{*}{ Day } & CFS (\%) & \multicolumn{4}{|c|}{ CFS } \\
\hline & & Total LAB & Lactococcus & Labmix & S. thermophilus \\
\hline \multirow[t]{4}{*}{0} & 0 & \multicolumn{4}{|c|}{$1.38 \times 10^{6} \pm 2.56 \times 10^{4}$} \\
\hline & 30 & \multicolumn{4}{|c|}{$1.45 \times 10^{6} \pm 1.06 \times 10^{4}$} \\
\hline & 40 & \multicolumn{4}{|c|}{$1.88 \times 10^{6} \pm 1.52 \times 10^{4}$} \\
\hline & 50 & \multicolumn{4}{|c|}{$1.68 \times 10^{6} \pm 2.82 \times 10^{4}$} \\
\hline \multirow[t]{4}{*}{1.5} & 0 & \multicolumn{4}{|c|}{$1.28 \times 10^{9} \pm 7.03 \times 10^{8}$} \\
\hline & 30 & $3.35 \times 10^{8} \pm 3.53 \times 10^{7^{*}}$ & $8.50 \times 10^{8} \pm 7.07 \times 10^{7 * *}$ & $2.05 \times 10^{8} \pm 7.07 \times 10^{6^{*}}$ & $4.40 \times 10^{8} \pm 8.48 \times 10^{7 * *}$ \\
\hline & 40 & ND & $1.55 \times 10^{8} \pm 3.53 \times 10^{7^{*}}$ & $4.15 \times 10^{8} \pm 7.77 \times 10^{7^{*}}$ & $5.80 \times 10^{8} \pm 2.82 \times 10^{7 * *}$ \\
\hline & 50 & $5.25 \times 10^{8} \pm 7.07 \times 10^{6^{* *}}$ & $8.50 \times 10^{8} \pm 7.07 \times 10^{6^{* *}}$ & $2.90 \times 10^{8} \pm 1.41 \times 10^{7^{*}}$ & $2.55 \times 10^{8} \pm 6.36 \times 10^{7^{*}}$ \\
\hline \multicolumn{6}{|c|}{ B. S. aureus } \\
\hline \multirow[t]{2}{*}{ Day } & CFS (\%) & \multicolumn{4}{|c|}{ CFS } \\
\hline & & Total LAB & Lactococcus & Labmix & S. thermophilus \\
\hline \multirow[t]{3}{*}{0} & 0 & \multicolumn{4}{|c|}{$4.30 \times 10^{6} \pm 1.76 \times 10^{4}$} \\
\hline & 20 & \multicolumn{4}{|c|}{$3.57 \times 10^{6} \pm 3.53 \times 10^{4}$} \\
\hline & 25 & \multicolumn{4}{|c|}{$3.41 \times 10^{6} \pm 3.53 \times 10^{4}$} \\
\hline \multirow[t]{3}{*}{1.5} & 0 & \multicolumn{4}{|c|}{$2.20 \times 10^{8} \pm 4.24 \times 10^{7}$} \\
\hline & 20 & $8.50 \times 10^{7} \pm 7.07 \times 10^{6^{*}}$ & $8.60 \times 10^{8} \pm 8.48 \times 10^{7 * *}$ & $2.85 \times 10^{8} \pm 2.12 \times 10^{7 * *}$ & $2.15 \times 10^{8} \pm 7.07 \times 10^{6 * *}$ \\
\hline & 25 & $2.20 \times 10^{8} \pm 8.48 \times 10^{7 * *}$ & $7.50 \times 10^{7} \pm 3.53 \times 10^{7^{*}}$ & $5.00 \times 10^{7} \pm 0.0^{*}$ & $9.00 \times 10^{7} \pm 2.82 \times 10^{7^{*}}$ \\
\hline \multicolumn{6}{|c|}{ C. Salmonella Ml1 } \\
\hline \multirow[t]{2}{*}{ Day } & CFS (\%) & \multicolumn{4}{|c|}{ CFS } \\
\hline & & Total LAB & Lactococcus & Labmix & S. thermophilus \\
\hline \multirow[t]{3}{*}{0} & 0 & \multicolumn{4}{|c|}{$1.55 \times 10^{6} \pm 4.24 \times 10^{4}$} \\
\hline & 20 & \multicolumn{4}{|c|}{$1.75 \times 10^{6} \pm 1.06 \times 10^{4}$} \\
\hline & 25 & \multicolumn{4}{|c|}{$1.56 \times 10^{6} \pm 2.82 \times 10^{4}$} \\
\hline \multirow[t]{3}{*}{1.5} & 0 & \multicolumn{4}{|c|}{$5.90 \times 10^{8} \pm 3.39 \times 10^{7}$} \\
\hline & 20 & $2.00 \times 10^{7} \pm 1.41 \times 10^{7^{*}}$ & $5.00 \times 10^{7} \pm 1.41 \times 10^{7^{*}}$ & $2.00 \times 10^{7} \pm 1.41 \times 10^{7^{*}}$ & $2.50 \times 10^{7} \pm 7.07 \times 10^{6^{*}}$ \\
\hline & 25 & ND & $4.50 \times 10^{7} \pm 2.12 \times 10^{7^{*}}$ & $2.00 \times 10^{7} \pm 1.41 \times 10^{7^{*}}$ & $2.50 \times 10^{7} \pm 7.07 \times 10^{6^{*}}$ \\
\hline
\end{tabular}

For each bacterium, those experimental CFUs that are significantly less than the respective day 1.5 control CFU are marked with ( ${ }^{*}$ ), while those that are not are marked with $\left(^{* *}\right)$. ND- not done.

this work is not explainable as having been mediated by low $\mathrm{pH}$ alone as most of the 17 CFSs tested before $\mathrm{pH}$ adjustment were not effective. This ascribes the inhibitory effect largely, if not totally, to non-bacteriocin metabolites secreted by the LAB isolates into the growth medium. It is possible that there was a combined synergistic effect between low $\mathrm{pH}$ and the metabolites. Biochemical characterization of the substances is needed to ascertain their exact nature.

The effects of the CFSs in inhibiting the bacterial pathogens were transitory, as was also seen in the work of others $[34,35]$. Colonies from experimental cultures were both fewer in numbers and much smaller in size. Upon transfer of samples from apparently completely inhibited cultures to fresh broth medium, however, growth occurred. After a second serial transfer, the resulting colonies attained comparable sizes to those from control cultures, indicating some bacterial populations remained viable but not culture-detectable.

\section{Conclusions}

Pathogens such as L. monocytogenes with tolerance or resistance to common food preservation methods and disinfectants, exceptional adaptation to changing environments and persistence in food-processing facilities continue to pose risk to human health. The antibiotic arsenal is limited, and bacterial pathogens often develop resistance to newly introduced antibiotics. These facts dictate that, along with new antibiotics, suitable pathogen control alternatives that are safe and efficacious are needed. LAB are suitable candidates in the search for these alternatives, and globally, some are already used in 
probiotic formulations. In this work, it was demonstrably possible to significantly retard the growth of the tested bacteria by LAB metabolites. To the best of our knowledge, we are not aware of reports of studies on local fermented foods that simultaneously identified the LAB species and pathogenic bacteria within the specific fermented foods and studied the interactions between the two groups of bacteria. Similar other studies have been done elsewhere, but they do not necessarily apply universally. Even members of the same bacterial species can have contrasting virulence characters (an example would be the Enterococci: Enterococcus faecalis, a probiotic strain and a pathogenic clinical isolate of Enterococcus faecalis).

This study was limited in scope, but we intend to further this work by focusing not only on the antimicrobial properties of the LAB, but also isolation and identification of the metabolites, their mechanisms of action, suitable modes of application, other potential applications, safety issues including the presence of virulence factors and transferable antibiotic resistance and comparison to standard type strains. In addition, a medium-to-long term goal would be to promote and advance probiotic science in our setting, which is virtually unknown at this time. For this, full characterization of the resident microbial flora within fermented foods and drinks of local origin is an imperative.

\section{Abbreviations \\ LAB: Lactic acid bacteria; CFS: Cell-free supernatant; MRS: Mann, Rogossa, Sharpe; CFU: Colony-forming units; TSB: Tryptic soy broth; LB: Luria-Bertani.}

\section{Competing interests}

The authors declare that they have no competing interests.

\section{Authors' contributions}

SHM, NZ, TT, EA and NE conducted the experiments. SHM analyzed the data and wrote the manuscript. AA reviewed the manuscript. SHM and AA conceived of the study and participated in its design. All authors read and approved the final manuscript.

\section{Acknowledgements}

This work was supported by fund from the AHRI core budget. We thank Tsehaynesh Lemma and Selam Jemberu for supplying some of the type strains and technical assistance. We thank Rawleigh Howe for reading and commenting on the manuscript.

\section{Author details}

${ }^{1}$ Aklilu Lemma Institute of Pathobiology, Addis Ababa University, Addis Ababa, Ethiopia. ${ }^{2}$ Armauer Hansen Research Institute, P.O. Box 1005, Addis Ababa, Ethiopia. ${ }^{3}$ Debre Berhan University, Debre Berhan, Ethiopia.

\section{Received: 2 August 2014 Accepted: 11 August 2014}

Published: 4 September 2014

\section{References}

1. Hoffmann S, Batz MB, Morris JG: Annual cost of illness and quality-adjusted life year losses in the United States due to 14 foodborne pathogens. J Food Prot 2012, 75:1292-1302.

2. Scharff RL: Economic burden from health losses due to foodborne illness in the United States. J Food Prot 2012, 75:123-131.

3. Food Safety: Centers for disease control and prevention. www.cdc.gov/ foodsafety/facts.html.
4. Pan Y, Breidt F, Kathariou S: Resistance of Listeria monocytogenes biofilms to sanitizing agents in a simulated food processing environment. Appl Environ Microbiol 2006, 72:7711-7717.

5. Müller A, Rychli K, Muhterem-Uyar M, Zaiser A, Stessl B, Guinane CM, Cotter PD, Wagner M, Schmitz-Esser S: Tn6188 - a novel transposon in Listeria monocytogenes responsible for tolerance to benzalkonium chloride. PLoS One 2013, 8:e76835.

6. Vázquez-Boland JA, Kuhn M, Berche P, Chakraborty T, Domínguez-Bernal G, Goebel W, González-Zorn B, Wehland J, Kreft J: Listeria pathogenesis and molecular virulence determinants. Clin Microbiol Rev 2001, 14:584-640.

7. Camejo A, Carvalho F, Reis O, Elsa Leitão E, Sousa S, Cabanes D: The arsenal of virulence factors deployed by Listeria monocytogenes to promote its cell infection cycle. Virulence 2011, 2:379-394.

8. Abee T, Krockel L, Hill C: Bacteriocins: modes of action and potentials in food preservation and control of food poisoning. Int J Food Microbiol 1995, 28:169-185.

9. Caplicec E, Fitzgerald GF: Food fermentations: role of microorganisms in food production and preservation. Int J Food Microbiol 1999 50:131-149.

10. Natrajan N, Sheldon BW: Inhibition of Salmonella on poultry skin using protein- and polysaccharide-based films containing a nisin formulation. J Food Prot 2000, 63:1268-1272

11. Benkerroum N, Oubel H, Sandine WE: Effect of nisin on yogurt starter, and on growth and survival of Listeria monocytogenes during fermentation and storage of yogurt. Int J Food Saf 2003, 1:1-5.

12. De Vuyst L, Leroy F: Bacteriocins from lactic acid bacteria: production, purification, and food applications. J Mol Microbiol Biotechnol 2007, 13:194-199.

13. Atassi F, Servin AL: Individual and co-operative roles of lactic acid and hydrogen peroxide in the killing activity of enteric strain Lactobacillus johnsonii NCC933 and vaginal strain Lactobacillus gasseri KS120.1 against enteric, uropathogenic and vaginosis-associated pathogens. FEMS Microbiol Lett 2010, 304:29-38.

14. O'Shea EF, Cotter PD, Ross RP, Hill C: Strategies to improve the bacteriocin protection provided by lactic acid bacteria. Curr Opin Biotechnol 2013, 24:130-134.

15. Dabour N, Zihler A, Kheadr E, Lacroix C, Fliss I: In vivo study on the effectiveness of pediocin PA-1 and Pediococcus acidilactici UL5 at inhibiting Listeria monocytogenes. Int J Food Microbiol 2009, 133:225-233.

16. Riley MA, Robinson SM, Roy CM, Dennis M, Liu V, Dorit RL: Resistance is futile: the bacteriocin model for addressing the antibiotic resistance challenge. Biochem Soc Trans 2012, 40:1438-1442.

17. Goh Y-L, He H, March JC: Engineering commensal bacteria for prophylaxis against infection. Curr Opin Biotechnol 2012, 23:924-930.

18. Wells J: Mucosal vaccination and therapy with genetically modified lactic acid bacteria. Ann Rev Food Sci Technol 2011, 2:423-445.

19. Koo OK, Amalaradjou MAR, Bhunia AK: Recombinant probiotic expressing Listeria adhesion protein attenuates Listeria monocytogenes virulence in vitro. Plos One 2012, 7:e29277.

20. Cotter PD, Ross RP, Hill C: Bacteriocins - a viable alternative to antibiotics? Nat Rev Microbiol 2013, 11:95-105.

21. Joint Food and Agriculture Organization of the United Nations/World Health Organization: Working Group Report on Drafting Guidelines for the Evaluation of Probiotics in Food, London, Ontario, Canada. April 30 and May, 2002 [cited 2010 Aug 25]. ftp://ftp.fao.org/es/esn/food/wgreport2.pdf.

22. MacFadden RR: Biochemical Tests for Identification of Medical Bacteria. 3rd edition. Philadelphia: Lippincott Williams and Wilkins; 2000.

23. Murray PR, Shea Y: Guide to Clinical Microbiology. 3rd edition. Washington, DC: American Society for Microbiology; 2004.

24. Sambrook J, Fritsch EF, Maniatis T: Molecular Cloning: A Laboratory Manual. Cold Spring Harbor Laboratory: New York; 1989.

25. Cleveland J, Montville TJ, Nes IF, Chikindas ML: Bacteriocins: safe, natural antimicrobials for food preservation. Int J Food Microbiol 2001, 71:1-20.

26. Cotter PD, Hill C, Ross RP: BACTERIOCINS: developing innate immunity for food. Nat Rev Microbiol 2005, 3:777-788.

27. Jack RW, Tagg JR, Ray B: Bacteriocins of gram-positive bacteria. Microbiol $\operatorname{Rev} 1995$, 59:171-200.

28. Mélançon D, Grenier D: Production and properties of bacteriocin-like inhibitory substances from the swine pathogen Streptococcus suis serotype 2. Appl Environ Microbiol 2003, 69:4482-4488. 
29. Walls T, Power D, Tagg J: Bacteriocin-like inhibitory substance (BLIS) production by the normal flora of the nasopharynx: potential to protect against otitis media? J Med Microbiol 2003, 52:829-833.

30. Gálvez A, Abriouel H, Benomar N, Lucas R: Microbial antagonists to foodborne pathogens and biocontrol. Curr Opin Biotechnol 2010, 21:142-148.

31. Rodríguez JM, Martínez MI, Suárez AM, Martínez JM, Hernández PE: Research note: unsuitability of the MRS medium for the screening of hydrogen peroxide-producing lactic acid bacteria. Lett App/ Microbio/ 1997, 25:73-74.

32. Pridmore RD, Pittet AC, Praplan F, Cavadini C: Hydrogen peroxide production by Lactobacillus johnsonii NCC 533 and its role in antiSalmonella activity. FEMS Microbiol Lett 2008, 283:210-215.

33. Bleicher A, Stark T, Hofmann T, Bogovic Matijasić B, Rogelj I, Scherer S, Neuhaus K: Potent antilisterial cell-free supernatants produced by complex red-smear cheese microbial consortia. J Dairy Sci 2010, 93:4497-4505

34. Bouttefroy A, Millie're J-B: Nisin-curvaticin 13 combinations for avoiding the regrowth of bacteriocin resistant cells of Listeria monocytogenes ATCC 15313. Int J Food Microbiol 2000, 62:65-75.

35. Hartmann HA, Wilke T, Erdmann R: Efficacy of bacteriocin-containing cell-free culture supernatants from lactic acid bacteria to control Listeria monocytogenes in food. Int J Food Microbiol 2011, 146:192-199.

doi:10.1186/1756-0500-7-606

Cite this article as: Mariam et al:: Potential of cell-free supernatants from cultures of selected lactic acid bacteria and yeast obtained from local fermented foods as inhibitors of Listeria monocytogenes, Salmonella spp. and Staphylococcus aureus. BMC Research Notes 2014 7:606.

\section{Submit your next manuscript to BioMed Central and take full advantage of:}

- Convenient online submission

- Thorough peer review

- No space constraints or color figure charges

- Immediate publication on acceptance

- Inclusion in PubMed, CAS, Scopus and Google Scholar

- Research which is freely available for redistribution 